\title{
Alessandro Chidichimo* \\ Une source du premier cours de linguistique générale de Saussure, octobre 1906
}

DOI 10.1515/sem-2016-0073

\begin{abstract}
Dans cet article, je publie un texte écrit par Ferdinand de Saussure en octobre 1906 et correspondant à une proposition de programme pour le premier cours de linguistique générale qui commença le 16 janvier 1907. Ce texte a été présenté aux séances de la Faculté de Lettres et Sciences sociales de l'Université de Genève à l'occasion des discussions à propos de la succession de Saussure sur la poste d'enseignement de linguistique générale de Joseph Wertheimer. Ce document constitue la première source saussurienne confirmée de ce premier cours et s'achève, en outre, sur quelques lignes décrivant ce qui sera un deuxième cours. L'histoire saussurienne raconte, en fait, que des trois cours de linguistique générale de Saussure (1907-1911), nous ne disposons de notes manuscrites de Saussure lui-même que pour les deux derniers cours. Je reconstruis donc l'histoire du début des cours saussuriens qui furent la source du Cours de linguistique générale et je publie le document avec des annotations inhérentes à l'histoire et à la philologie saussurienne. Cette introduction au premier cours doit être ajoutée à toutes les autres sources inhérentes au Cours de linguistique générale.
\end{abstract}

Keywords: Saussure, linguistique générale, manuscrits, Premier cours, philologie

\section{Introduction}

L'histoire saussurienne raconte que des trois cours de linguistique générale de Ferdinand de Saussure donnés entre le 16 janvier 1907 et le 4 juillet 1911, nous ne disposons de notes écrites de la main de Saussure que pour les deux derniers cours. ${ }^{1}$ Pour ce qui concerne le premier cours, il n'y a qu'une hypothèse de

1 Il s'agit des notes du deuxième et troisième cours BGE Ms.fr. 3951/22-23, CLG/E 3331-3340, et AdS 372, p. 241-254 (sauf p. 244, faisant apparemment partie de la recherche sur les Anagrammes) publiés d'abord en ELG et puis in Saussure (2011).

*Corresponding author: Alessandro Chidichimo, Université de Genève, 1205 Geneva, Switzerland, E-mail: alessandrochidichimo@gmail.com 
source manuscrite de Saussure (cf. BGE Ms.Fr. 3951/20), formulée d'abord par Robert Godel (1902-1984; SM: 13, 51; Godel 1960: 8) ${ }^{2}$ et reprise ensuite par Rudolf Engler (1930-2003; CLG/E 3329, ELG: 297). ${ }^{3}$ Les notes de ce cours n'ont pas été utilisées en entier par Bally et Sechehaye pour nourrir l'édition du texte qui sera le CLG et ils les ont reléguées dans des parties marginales du texte (cf. CLG/D: 320).

Dans les Archives de l'Université de Genève [AUG] se trouve un document correspondant à une proposition de programme pour le premier cours, délivrée par Saussure lui-même et qui constitue la première source saussurienne confirmée de ce même cours. ${ }^{4}$ Ce document s'achève en outre sur quelques lignes décrivant ce qui serait un deuxième cours, hypothétique au moment où Saussure écrivait. Dans les pages suivantes, je reconstruis l'histoire de la genèse et son contexte philologique et je publie ce document. Ce texte pour le premier cours doit être ajoutée aux sources du CLG.

\section{La linguistique générale à Genève avant Saussure: Joseph Wertheimer (1833-1908)}

Le prédécesseur de Saussure à la chaire genevoise de linguistique générale, Joseph Wertheimer, ${ }^{5}$ n'était pas seulement professeur à l'université, mais il fut d'abord le grand rabbinde Genève. D’origine française alsacienne, Wertheimer arrive à Genève en 1859, après des études à Paris, Strasbourg et pourvu d'un doctorat en théologie de Metz (1857), pour devenir le rabbin de la synagogue genevoise qui venait d'être inaugurée (Plançon 2008: 14-15 NOT REFERENCED). Si de 1869 à 1873 "le cours avait été donné par Krauss, ${ }^{6}$ titulaire de la chaire de

2 Godel montre ses doutes: "Le début: Dans la vie du langage: I Les Évolutions I. Le changement phonétique 2 ... rappelle le plan du cours I; mais la suite ne s'y retrouve pas” (SM 51).

3 Les étudiants présents au premier cours étaient: A. Alexandroff, Henri Chavannes, George Turner Ford, Albert Riedlinger, Marie Wittmann, Louis Caille (Linda 2001: 175; Joseph 2012: 492). Cf. Vincent (2010 et 2014) à propos des notes prises par Louis Caille et leur transcription. 4 Le mérite d'avoir mis en lumière cette source des AUG, ignorée auparavant en tant que possible source de documentation saussurienne, revient à Mme Fabienne Reboul, que je remercie de m'avoir indiqué cette piste de recherche. Mes remerciements vont aussi à Mme Dominique Torrione des AUG et à tous les collaborateurs des archives.

5 À propos de Wertheimer cf. Plançon 2001 NOT REFERENCED. Dans le cadre du lien avec Saussure cf. Zwanziger (1981; Joseph 2012; Fryba-Réber 2013).

6 Hermann Krauss (1825-1889) fut professeur d'allemand de Saussure à Genève durant sa fréquentation du gymnase (cf. Joseph 2012: 143). Joseph reporte le nom Carl Krauss, mais il s'agit de la même personne signalée par Fryba-Réber (2013: 262 et 299), qui propose le nom complet Hermann-Oscar-Carl Krauss. Saussure était une des personnes envisagées pour 
langue et littérature allemandes, sous le titre de Philologie, puis de Linguistique comparée" (SM: 29 n.61), à partir de 1873 Wertheimer sera chargé de cours, puisen 1874 professeur de Linguistique et philologie. ${ }^{7}$ C'est à cette occasion que paraît la première et seule publication linguistique de Wertheimer dont nous ayons connaissance. Il s'agit del'opuscule La linguistique Discours prononcé le 30 octobre 1877 à l'ouverture du cours de linguistique. On retrouve dans ce texte plusieurs références à De la forme et de la fonction des mots par Michel Bréal (1832-1915; Bréal 1866), alsacien et d'origine juive comme Wertheimer lui-même, et tout le texte est d'inspiration bréalienne (cf. SM: 29, n.23; Zwanziger1981). ${ }^{8}$ Aucune autre publication de Wertheimer sur ce sujet n'est connue. ${ }^{9} \mathrm{Si}$ on interrogeait les diverses archives en espérant retrouver ses manuscrits personnels, la situation serait décevante. ${ }^{10}$ À part un lot de sa correspondance, répartie entre la BGE, les archives d'État de Genève et les autres lettres qui sont probablement présentes dans les archives de ses correspondants, il existe un dossier

succéder à Krauss à la chaire de langues et littératures germaniques à l'Université de Genève en 1889 (cf. Reboul 2010).

7 "La Philologie comparée fut demandée à l'Alsacien Joseph Wertheimer, grand rabbin de Genève depuis 1859. Il fut chargé d'un cours de linguistique par arrêté du 26 septembre 1873, puis nommé professeur de Linguistique et de Philologie en 1874” (Borgeaud 1934: 469).

8 D'après ce que nous avons pu vérifier, il n'existe pas d'échanges entre Bréal et Wertheimer. Il faut supposer que les deux acteurs se soient rencontrés en 1894 à Genève, lors du Colloque des orientalistes où Bréal fut présent et dont Wertheimer faisait partie du Comité d'organisation. Saussure, avec Paul Oltramare (1854-1930), était le secrétaire du colloque. Bréal fut le professeur de Saussure à l'École des Hautes Études avant de lui conférer des enseignements toujours à l'École.

9 Ses publications principales sont Wertheimer (1865, 1880). Dans les archives du Journal de Genève [JdG] on retrouve en outre une conférence de Wertheimer sur l'origine des langues en date 5 mars 1891 (JdG, 5 mars 1891: 5). Et on apprend que, dans le cadre des cours publics à l'Aula de l'Université de Genève, Wertheimer donna dix séances sur les Védas du 5 janvier au 7 février 1874 (JdG, 29 octobre 1873: 3). Ce même Journal cite, lors de la démission de Wertheimer en 1906, le volume Introduction à l'étude de la linguistique, mais qui est en fait La linguistique de 1877 (JdG, 5 septembre 1906: 2).Wertheimer sera dans le comité d'honneur du Congrès Universel de l'esperanto qui eut lieu à Genève entre le 27 août et le 5 septembre 1906 (JdG, 27 août 1906: 2). Il faut rappeler que le frère de Saussure, le mathématicien René de Saussure (1868-1943) était à Genève durant ce colloque et qu'il était un espérantiste très actif publiant sous le nom d'Antido.

10 J'ai essayé de retrouver d'autres manuscrits de Wertheimer et des notes des étudiants en m'adressant tant au Département des manuscrits de la Bibliothèque de Genève [BGE] qu'aux AUG. J'ai également essayé de retrouver ses descendants en France (qui auraient pu garder des documents) en m'adressant au Consistoire Général de Paris, mais ma recherche n'a pas abouti. Enfin, j'ai consulté M. Plançon, auteur d'une monographie sur Wertheimer, et divers membres de la communauté juive et du rabbinat genevois, mais cette fois encore, pour l'instant, il n'y a pas eu de découvertes de nouvelles sources. 
conservé, toujours à la BGE, détenant les notes prises durant les cours de plusieurs professeurs de l'Université de Genève, y compris Wertheimer, remontant aux années académiques 1878-1879 et 1880-1881 (201-346). ${ }^{11}$ Il s'agit d'un volume relié et il est indiqué que les notes se référent aux cours de Philologie $d u$ grec et Philologie de l'hébreu. De plus, il y a un nombre mineur de pages consacrées à la linguistique (338-345), mais qui ne sont pas signalées par les archives (cf. Joseph 2012: 489). Au tout début de ces pages sur la linguistique, Wertheimer introduit une différenciation entre la phonologie comme "étude du mot en tant que sens" et la morphologie, "c'est l'étude de la forme du mot" (BGE Cours Univ. 778: 338). ${ }^{12}$ Par la suite, Wertheimer considère la description de diverses familles de langues et des possibles classifications. Dans le manuscrit, l'élève qui a pris les notes a reporté des extraits tirés du Discours sur les rapports entre la science et la religion révélée par le cardinal Nicholas Wiseman (Wiseman 1838), donc peut-être ${ }^{13}$ une des sources utilisées par Wertheimer durant ses cours (BGE Cours Univ. 778: 342). ${ }^{14}$ Les programmes des cours de Wertheimer donnent encore d'autres informations. Si on considère les derniers cours donnés juste avant le passage à Saussure, on retrouve pour le Semestred'hiver de 19051906: pour l'enseignement de linguistique: phonologie, morphologie, racines,

11 BGE Cours Univ. 778, notes prises par L.B. - l'auteur n'a pas été identifié par les archives. Le document est folioté en numérotant les pages et non les feuilles.

12 Pour la terminologie utilisée par Saussure pour la phonétique et la phonologie cf. Bergonioux 2010: 106-109. En ce qui concerne la phonologie dans le premier cours cf. Joseph (2012: 495). Pour la définition de la morphologie, on peut citer, entre autres, le manuscrit $D e$ l'essence double du langage [ED] (BGE AdS 372, p. 35). Et de même, en ce qui concerne la distinction entre phonétique et morphologie (BGE AdS 372, p. 97). La morphologie sera regroupée sous la sémiologieen opposition à la phonétique: "(Sémiologie = morphologie, grammaire, Syntaxe, Synonymie, Rhétorique, stylistique, lexicologie etc... . le tout étant inséparable)/Phonétique” (BGE AdS 372, p. 69). Enfin, la morphologie correspond à l'étude conduite dans un état de langue: “[284] Thèses (1) Il n’y a de morphologie que dans un état de langue donné; mais (2) tout c'est qui est à étudier dans un état de langue donné est de la morphologie" (BGE Ms.fr. 3953, f. 284).

13 Nous ne savons pas si ces références à Wiseman annotées par cet élève pourraient être indépendantes du cours de Wertheimer.

14 Nicholas Wiseman (1802-1865), cardinal, docteur en théologie et professeur de langues orientales à l'université de Rome (http://www.britannica.com/biography/Nicholas-Wiseman). Le volume de Wiseman s'ouvre avec deux discours Sur l'étude comparative des langues (25-140), la première partie du premier discours porte le titre d'Ethnographie, ou étude comparative des langues. Les passages reportés par l'élève de Wertheimer portent sur la définition d'ethnographie justement comme reporté dans le titre "étude comparative des langues",laquelle correspond à une "classification des nations d'après l'étude comparé des langues" (Wiseman 1838: 31). Cet article n'est pas le lieu pour discuter d'une comparaison entre ces notes, le texte de Wiseman et leurs divergences avec Saussure. 
flexions, particules; pour le Semestre d'été: mythologie sémitique. Pour l'année suivante, 1906-1907, quand Saussure lui succédera, le Semestre d'hiver prévoyait le même programme que l'année précédente (AUG, Programme des cours de l'Université de Genève, 1891-1906). Si on peut penser que durant les plus de trente années de cours donnés par Wertheimer en linguistique générale, il y a eu des mises à jour des programmes et des contenus des cours, on constate qu'il proposait un point de vue différent en terme d'horizons de connaissances et d'actualité des programmes de ce qui constituera les cours qui suivront avec la prise en charge de la linguistique par Saussure.

\section{Le rapport entre Saussure et Wertheimer et l'enseignement de linguistique générale $(1891,1906)$}

L'histoire de la relation entre Saussure et Wertheimer commence bien avant 1906. Saussure, durant sa fréquentation de l'Université de Genève en tant qu'étudiant entre1875 et 1876,a évité, selon Zwanziger (1981), les cours de Wertheimer. Si l'affirmation de Zwanziger ne semble être soutenue par aucun document, Joseph (2012) a utilisé plus de prudence en considérant le possible gain que Saussure aurait pu avoir à suivre les cours de Wertheimer grâce à l'intérêt de ce dernier pour Whitney, un des auteurs de référence de Saussure: "So it is unclear whether his ignoring Wertheimer was the 'good instinct' described by De Mauro, or a missed opportunity to advance his understanding about language" (Joseph 2012: 173). ${ }^{15}$ Ensuite, quand la possibilité d'une nomination de Saussure à l'Université de Genève en 1891 en tant que professeur se présente, elle n'est pas acceptée facilement par la majorité des autres professeurs de la Faculté et, en particulier, un des opposants principaux est Wertheimer. ${ }^{16}$ Les registres des séances de la Faculté montrent la résistance à la création de la chaire de Saussure. Les problèmes discutés portent en premier lieu, comme on vient de le dire, sur la présence de Wertheimer à la chaire de

15 À propos de la période durant laquelle les deux seront collègues, Zwanziger a reporté le témoignage de Léopold Gautier (1884-1973) à travers Godel: "Léopold Gautier m’a raconté qu'aux épreuves orales Saussure, coexaminateur, écoutait sans broncher, impassible et impénétrable, les questions saugrenues de son collègue” (Zwanziger 1981: 43; Fryba-Réber 2013: 276). Ce témoignage n'a pas une valeur explicative, mais plus que de décrire un jugement explicite de Saussure, il montre le sentiment de l'entourage saussurien par rapport à Wertheimer.

16 À propos de la création de la chaire de Saussure en 1891 et du contexte politique et académique cf. Reboul (2010). 
Linguistique et philologie et par conséquent sur la différenciation à faire entre les deux chaires, enfin, sur la nécessité réelle de la création d'une nouvelle chaire. Ce dernier argument est soutenu par les professeurs opposés à la chaire de Saussure au motif qu'il y a un manque d'étudiants pour un enseignement comme celui de Saussure. ${ }^{17}$ Dans cette situation, une discussion très vive a lieu à la Faculté. Wertheimer ne demande pas seulement de la clarté sur la différenciation entre sa chaire et celle de Saussure, mais le 15 juin 1891, il en arrive à demander que le préavis sur la création de la chaire de Grammaire comparée des langues indoeuropéennes destinée à Saussure et proposée par le Département de l'instruction publique [DIP] soit refusé par la Faculté. ${ }^{18}$ La proposition de Wertheimer est tout de suite soutenue par la quasi-totalité des autres professeurs. ${ }^{19}$ Et l'argument principal de la discussion devient bientôt une question de personne: "(-)voulons-nous oui ou non appeler M. F. de Saussure à la Faculté ?” La proposition de Jules Nicole (18421921), ${ }^{20}$ qui propose une négociation directe entre Wertheimer et le DIP avant tout

17 La pénurie d'étudiants pour des cours comme le sanscrit, par exemple, dispensé par Saussure, sera un des arguments des attaques faites contre lui durant la période de la discussion à Genève de la réforme de l'université en 1912 (cf. Joseph 2008).

18 Il existait deux possibilités en matière de création de chaires: a) la Faculté concernée en prenait l'initiative, s'assurait de l'accord de l'Université et demandait l'avis du DIP - en cas d'accord de celui-ci, la décision finale appartenait au Grand Conseil (le corps législatif de la République et canton de Genève); b) le DIP proposait à la Faculté concernée la création d'une chaire sur une matière, la Faculté donnait son préavis à cet égard, préavis qui devait être confirmé par l'Université et le DIP décidait ensuite de soumettre ou non la proposition au Grand Conseil. Voici donc pourquoi Wertheimer peut proposer à la Faculté de refuser la proposition de préavis par le DIP:

M. Wertheimer considère l'enseignement proposé comme rentrant dans le sien. Qui dit grammaire comparée dit linguistique. Il ne saurait consentir à cette création, sur laquelle il s'étonne de n'avoir pas été consulté. Pour profiter d'un cours comme celui-là il faut des auditeurs, qui connaissent le sanscrit. Parmi nos étudiants il n'y en pas. Pendant plusieurs semestres un privat-docent fort distingué a donné sans succès un cours de sanscrit. Il prie la faculté de ne pas approuver la création d'une chaire qui faisait double emploi avec la sienne. (AUG/RS, 1984/20/89, 15 juin 1891, publié in Reboul 2010: 238)

19 Les professeurs Pierre Vaucher (1833-1898), Louis Jaquemot (1844-?), Ernest Strohelin (1844-1907), Édouard Rod (1857-1910) interviennent d'une manière ou d'une autre contre la création d'une nouvelle chaire. On découvre bizarrement que la sauvegarde du status quo et l'allergie typiquement académique aux changements n'est pas une invention moderne.

20 Nicole était le professeur de langue et littérature grecque. Saussure sera son suppléant durant le semestre d'hiver de l'année académique 1896-1897 (AUG/RS 1894/20/90, 13 octobre 1896: 77, et 30 janvier 1897: 93) et puis encore une fois pour le semestre d'été de l'année académique 1898-1899 (cf. AUG/RS1894/20/90, 1 et 8 juin 1899, p. 223 et p. 225). Par rapport aux manuscrits de Saussure rédigés à l'occasion d'une de ces suppléances Mejia et RestrepoMontoya (2009: 181-182) ont fait l'hypothèse qu'il s'agissait du Semestre d'hiver 1896-97. Ils ont 
préavis de la Faculté, est adoptée à l'unanimité. Il souligne que “à la question de personne, le nom de M. Ferd. De Saussure est connu dans toute l'Europe. On s'étonne à Paris que Genève ne l'ait pas encore réclamé. Il lui paraît certain que la présence à Genève de $M$. de Saussure attirera des étudiants.” Bernard Bouvier abonde en ce sens. Enfin "la proposition de M. Nicole - renvoi au Département pour entente préalable avec M. Wertheimer - est adoptée à l'unanimité."21 Le 20 juin, les rencontres entre Wertheimer et le DIP semblent éclaircir la situation à l'avantage de Saussure. La Faculté exprime enfin un préavis favorable à sa chaire, mais seulement de façon extraordinaire et donc pour deux années (AUG/RS, 1984/ 20/89, 20 juin 1891, in Reboul 2010: 242-243). ${ }^{22}$ L'opinion de Wertheimer, donc, semble être changée lors de la séance du 22 juin du Sénat académique: "MM. De Crue, Nicole, Wertheimer parlent en faveur de la nouvelle chaire, en faisant valoir l'intérêt qu'il y a à former un ensemble de cours relatifs à la philologie et le mérite incontesté de la personne qui dans l'idée de la faculté devrait être appelée à cette chaire" (AUG, Registre des Séances du Sénat universitaire, 110a 2, 22 juin 1891). ${ }^{23}$ Le Sénat confirmera le préavis favorable donné par la Faculté à la création de la chaire extraordinaire de Saussure de Grammaire comparée des langues indo-européennes. ${ }^{24}$

publié aussi un passage d'une lettre d'Adrien Bovy, datant du 29 juin 1949 (BGE Ms.fr. 1599/5, f.8) adressée à Léop. Gautier, mais sans donner une datation de l'évènement dont il est question dans cette lettre. Dans leur texte Mejia et Restrepo-Montoya n'ont pas pris en compte que Saussure a remplacé deux fois Nicole. Bovy, je le crois, fait référence à la deuxième suppléance en 1899: “Au début d'un semestre d'été, $M$. Jules Nicole étant malade, il le suppléa pendant quelques semaines. Je me souviens de notre étonnement à la première leçon. Il s'agissait de lire Prométhée enchaîné, et nous n'avions pas dépassé le premier vers! Chaque mot avait eu son sort et ses titres de noblesse: Il nous semblait que Saussure inventait devant nous des langues inconnues" (BGE Ms.fr. 1599/5, f.8). Le 8 juin 1899, en effet, on peut lire que Saussure accepte de substituer Nicole et qu'il traitera le Prométhée: "D’accord avec M. Nicole, M. de Saussure a consenti à le remplacer et à interpréter, pendant le mois de juin, le 'Prométhée enchaîné,' d'Eschyle" (cf. AUG/RS1894/20/90, 8 juin 1899, p. 225).

21 Le même problème, la duplication ou superposition d'une chaire, est posé pendant la même séance par Jean-Jacques Gourd (1850-1909) par rapport à la chaire d'Esthétique et méthodologie de Adrien Naville. Dans ce cas, la solution adoptée fut également d'établir un contact direct entre le professeur Gourd et le DIP. Naville sera l'auteur de la classification des sciences (1901) où apparaîtra, pour la première fois, le terme sémiologie lié au nom de Saussure (Naville 1901: 103-106; CLG/D: 318-319, 331).

22 Mais encore sans obtenir l'unanimité (7 vœux contre 2). La négociation intervenue entre Wertheimer et le DIP n'empêche pas les professeurs Vaucher et Rod de souligner leur vœu contraire.

23 La référence à la philologie souligne le rôle prioritaire de l'enseignement de Wertheimer.

24 La difficulté de sa position et le rapport de son enseignement avec celui de Wertheimer sont bien clairs pour Saussure (cf. Saussure à Nicole, 16 juin 1891, BGE Ms.fr. 7925 in Reboul 2010: 240-242). 
Saussure deviendra professeur ordinaire en 1896. Àcette occasion, une première discussion a lieu le 5 mars 1896 (AUG/RS, 1984/20/90, 5 mars 1896: 41), inspirée par De Crue $^{25}$ qui prend l'initiative en proposant ce point à discuter, sans qu'il soit prévu par l'ordre du jour de la séance. La séance décisive sera tenue le 14 avril 1896, quand la Faculté donna un avis favorable à la transformation de la chaire extraordinaire en ordinaire. À cette occasion, Wertheimer parlera encore en faveur de Saussure: "M. Wertheimer se déclare très favorable à la proposition, à laquelle il a déjà donné sa signature. Il propose que le sanscrit soit mis en vedette dans le titre qui serait donné à la chaire nouvelle“ (AUG/RS, 1984/20/90, 14 avril 1896: 56-60). La proposition de mettre le sanscrit "en vedette" dans le titre, semble répondre encore une fois à l'exigence de maintenir la séparation entre le domaine de l'enseignement de Wertheimer et celui de Saussure. Au titre du cours de ce dernier, enfin, serait ajouté le sanscrit: Grammaire comparée des langues indoeuropéennes et sanscrit. ${ }^{26}$

Les rapports entre Wertheimer et Saussure et leurs chaires étaient loin d'être finis. En effet, lors du semestre d'été 1906, Saussure fut l'hypothétique suppléant pour le cours de linguistique de Wertheimer, tombé malade (cf. AUG/RS, 1984/20/91, 3 mars 1906). Mais cette suppléance n'eut jamais lieu, parce que Wertheimer an non ça pouvoir reprendreses leçons dès avril. Saussure prolongea, en parallèle, son congé commencé durant le semestre d'hiver, durant tout le semestre d'été. ${ }^{27}$ On peut imaginer que si cette éventualité avait eu lieu, Saussure aurait pu avoir l'occasion de préparer, par anticipation, son premier cours et d'arriver, ainsi, l'année académique suivante, plus prêt à entreprendre un nouvel enseignement.

Avant le début de l'année académique suivante, le premier septembre 1906, Wertheimer donne sa démission et Saussure fut finalement chargé de l'enseignement de Linguistique générale. ${ }^{28}$ Mais le chemin pour arriver à cette conclusion

25 Francis De Crue (1854-1928), professeur ordinaire d'archéologie, d'épigraphie et de paléographie, et puis d'histoire du Moyen-âge et d'histoire moderne (1898-1927; cf. FrybaRéber 2013: 278-279).

26 Cf. Saussure (2014: 246), lettre de remerciements de Saussure au Doyen de l'époque Eugène Ritter (1836-1928) datant le 16 avril 1896. Ritter, professeur d'histoire de la langue française et expert des manuscrits rousseauistes est une des personnalités qui contribuera à l'innovation de la Faculté de Lettres et Sciences sociales (cf. Fryba-Réber 2013). En outre, il sera un des correspondants de Bréal à propos des recherches de ce dernier sur Goethe (cf. Chidichimo 2013). 27 Le suppléant de Saussure sera encore Charles Bally (1865-1947), qu'était chargé de ce rôle déjà dans le semestre précèdent.

28 Cf. AUG/RS, 1984/20/91, 6 octobre 1906: 217-218; Bouvier (1907: 20). Le 18 septembre, à la suite de la séance du Conseil d'État de Genève, Wertheimer sera nommé Professeur honoraire. 
passait une fois encore par des discussions lors des séances de la Faculté. Le passage entre Wertheimer et Saussure s'insérait dans la réforme des deux licences en lettres et sciences sociales. Le 6 octobre 1906, la Faculté nomme une commission pour étudier l'avenir des enseignements de Wertheimer (cf. AUG/RS, 6 octobre 1906: 218). Saussure fait partie de cette commission avec Oltramare et Nicole. Au cœur de la réforme de la licence ès lettres, se trouve la réorganisation des enseignements de Wertheimer par rapport à la philologie et à la linguistique générale et il est demandé à Saussure d'établir un rapport sur ce sujet. ${ }^{29}$ Le rapport que Saussure rédige pour la réforme des enseignements est un possible programme du premier cours. On y trouve, de plus, des perspectives de développement de l'enseignement par rapport à un possible deuxième cours de linguistique générale (cf. AUG/RS, 27 octobre 1906: 220-223). Le texte de Saussure sera lu par Nicole et les autres professeurs présents à la séance donnèrent leur avis sur la note écrite par Saussure. À ce moment, le cours de Saussure n'a pas encore de titre officiel et on ne sait pas encore si celui de Wertheimer sera conservé, mais ce fut finalement Muret, et non Saussure, qui proposa le titre du nouvel enseignement de la chaire saussurienne:

Après quelques considérations complémentaires qu'expose M. de Saussure, et qui ont trait notamment aux rapports de la linguistique et des sciences sociales en général, M. Muret appuie les conclusions de la Commission.

M. de Girard marque l'intérêt du programme tracé par M. de Saussure pour les étudiants de sciences sociales. M. Bouvier informe la Faculté que le Bureau de l’Université se réunira lundi prochain 29 octobre, prendra connaissance des décisions de la Faculté touchant l'enseignement de la linguistique et examinera la question. Les conclusions de la commission, énoncées plus haut, sont acceptées. Muret propose comme titre du nouvel enseignement: Linguistique générale et grammaire comparée des langues indo-européennes (Approbations). ${ }^{30}$ M. Naville insiste sur l'avantage qu'il y a, pour arriver au but sans

29 Il est à souligner le fait que si les présences de Saussure lors de son arrivée à Genève depuis 1891 jusqu'au septembre 1906 ne sont pas vraiment assidues, depuis ce moment et pour toute la période jusqu'à la prise en charge du cours de linguistique, il sera constamment présent aux séances (cf. Chidichimo en préparation).

30 / Dans la séance suivante Muret affirme qu'il ne s'agissait que d'une suggestion: "M. Muret observe qu'il <a simplement proposé pour la partie de l'enseignement de M. Wertheimer qui doit être réunie à celui des langues indo-européennes le titre de linguistique générale, sans aucun changement au titre de l'enseignement actuel de M. de Saussure" (AUG/RS, 3 novembre 1906: 223). Cette déclaration de Muret, ancien élève de Saussure, nous met à l'abri d'une hypothèse qui voudrait la proposition de Muret faite avec l'appui de Saussure. De même, l'argument selon lequel Saussure aurait choisi un titre pareil n'est pas soutenu par les documents à disposition. Le titre du cours ne correspondait donc pas à un choix précis de Saussure par rapport à sa volonté de se débarrasser de la tradition précédente de la grammaire comparée ou pour des raisons de caractère théorique comme cela a été affirmé ailleurs. 
recourir à un changement de la loi, à parler d'enseignement, et non de chaire. (AUG/RS, 1984/20/91, 27 octobre 1906: 222-223).

Avant d'arriver à la reconnaissance officielle du cours, il restait à surmonter les difficultés de nature politico-administrative. En effet, afin de créer une nouvelle chaire, comme ce fut le cas pour Saussure en 1891, il fallait avoir l'approbation du DIP et ensuite du Conseil d'État, avec une procédure administrative et politique qui pouvait demander beaucoup de temps sans garantie de succès. Le choix est alors de ne pas créer une nouvelle chaire, mais de rajouter l'enseignement de linguistique générale à celui déjà donné par Saussure et de simplement changer le nom de la chaire de Saussure, qui deviendra Linguistique générale et grammaire comparée des langues indo-européennes. De même, la décision fut prise de ne pas créer un enseignement autonome de philologie et de demander à chaque professeur de la Faculté d'en donner des bases aux étudiants:

Après le décès de M. Joseph Wertheimer, en 1908, les "petits cours” de linguistique et de philologie qui composaient son enseignement ont été rattachés à d'autres chaires. La linguistique générale a été réunie à la grammaire comparée des langues indoeuropéennes et confiée à M. Ferdinand de Saussure, décédé en 1913, auquel a succédé M. Charles Bally. Il a été entendu, d'autre part, que les professeurs de langues et littératures, spécialement ceux de langues et littératures anciennes, donneraient à leurs étudiants les notions générales appartenant au domaine de la philologie. (Naville 1914: 84)

Si le bureau du Sénat Académique adopte à l'unanimité les propositions de la Faculté par rapport à l'enseignement de Wertheimer, il y aura encore des discussions relatives à l'enseignement et aux programmes des deux licences différentes. En résumé, si la proposition du programme du rapport de Saussure avait eu l'avis favorable, il fallait harmoniser le tout par rapport à l'organisation composite de la Faculté: “Une discussion s'engage sur les changements qui comporte, en ce qui concerne les programmes, la réorganisation de l'enseignement de la linguistique. Y prennent part MM. Muret, Naville, de Saussure, Oltramare” (cf. AUG/RS, 1984/20/91, 3 novembre 1906). Le Doyen Paul Duproix (1851-1912), professeur de pédagogie, propose de créer deux autres commissions pour étudier les changements à introduire dans les programmes et dans la procédure des examens des deux licences en lettre et en sciences sociales. Dans la première commission se trouvent Muret, Saussure, Nicole; dans la deuxième, A. Naville, de Girard, Saussure, Milhaud. Enfin, le travail et la stratégie choisie par la Faculté, après l'approbation du Sénat Académique le 3 novembre, seront gagnants et Le Journal de Genève annonce le 9 décembre 1906 la nouvelle des changements au sein de la Faculté, désormais acceptés aussi par le DIP. Et c'est à ce moment qui commence l'histoire des trois cours de linguistique générale de Saussure. 
Pour une évaluation correcte de ce premier cours de linguistique générale de Saussure, il y a donc une analyse contextuelle à faire. Dans l'étude des documents de Saussure, il faut toujours considérer le statut propre à chaque document et à chaque témoin. Nous avons vu que la discussion sur la réorganisation des cours était encore vivante pendant les séances suivantes pour adapter l'enseignement tant pour les étudiants de Lettres que pour les étudiants des Sciences Sociales. Comme nous l'avons vu, l'année académique était à son début, mais sans que le cours de linguistique générale soit assigné. Il fallait donc rapidement prendre une décision et prendre en considération les étudiants déjà inscrits, qui venaient, probablement, des années précédant la réforme de la licence et qui avaient la priorité. De plus, si le passage entre Wertheimer et Saussure s'insérait dans le cadre de la réforme de la licence ès lettres, donc durant une année de transition dans toute la Faculté, alors voici une explication partielle qui nous encourage à considérer la première année de l'enseignement de Saussure comme une année de passage. Difficile de ne pas faire l'hypothèse que le programme du premier cours de Saussure de1907, en tenant compte de sa responsabilité envers les étudiants, ne s'en est pas ressenti dans sa structure. Et, en effet, les registres des séances de la Faculté indiquent que le 21 janvier 1907 Muret et Saussure prirent en charge d'expliquer aux étudiants les changements apportés au programme de licence ès lettres (cf. AUG/RS, 1984/20/91, 12 décembre 1906: 239).On ajoute à cela le fait que Saussure, ayant manqué la possibilité de remplacer Wertheimer durant le semestre précédent (voir supra), n'avait pas vraiment eu la possibilité de se préparer pour le premier cours, étant donné la démission inattendue de son prédécesseur et le remplacement de Saussure qui n'était pas garanti: "In fact he withdrew only weeks before the start of the autumn 1906 semester, indicating that a sudden attack of ill health precipitated his retirement" (Joseph 2012: 492). On retrouve donc, dans le premier cours, l'empreinte saussurienne mais nous devons, en même temps, être prudent en ce qui concerne son évaluation générale:

The first course brought together much of Saussure's thinking about linguistic concepts and methods since his student days, material that had always been part of his teaching. At the same time it contained, in germ, the distinctive features of his later thought. The order is different from the familiar one of the published Course, and some ideas are less completely developed. Clearly he was feeling his way - but that is part of the appeal. (Joseph 2012: 492)

Chaque document saussuriena, en fait, besoin d'être placé dans le contexte de sa production matérielle, philologique et historique, donc en lien avec les autres sources manuscrites à disposition et avec les autres possibles témoignages à propos 
de la recherche saussurienne. Il n'y a pas de reconstruction possible sans ce travail préalable qui permet de mettre en lumière la continuité des recherches saussuriennes, l'épaisseur du contexte historique et qui permet d'aller plus loin dans la compréhension du lien entre philologie et théorie saussurienne.

\section{Le programme du premier cours de linguistique générale: description du document}

Les registres de la Faculté sont de grands volumes où les secrétaires des séances enregistraient les procès verbaux des séances de la Faculté de Lettres et Sciences sociales. La caractéristique majeure de ces documents est que les informations sont prises en partant des documents officiels avec une datation et une liste des personnes présentes. Les notes sont soumises à vérification et ratifiées par la Faculté. À chaque début de séance, le procès-verbal de la séance précédente était lu et approuvé ou corrigé si les professeurs participants à la séance enregistrée remarquaient des imprécisions. Le procès-verbal était, après cela, recopié dans le registre officiel des séances. Cette vérification ultérieure des informations par les principaux intéressés, et le statut officiel de ces documents, nous donne un degré de certitude et de fidélité sur la volonté de Saussure, par rapport au texte présenté ici. Plus encore, Saussure lui-même a rédigé le texte qui sera lu durant la séance par Jules Nicole. Le document, donc, a été recopié par le secrétaire de la séance, Edgard Milhaud (1873-1964) professeur d'Économie Politique, en partant du texte de Saussure. Les secrétaires des séances de la Faculté reportent, normalement, les discours et les prises de parole qui se passent entre les professeurs utilisant le discours indirect libre, ou bien en écrivant en troisième personne, par exemple: $M$. de Saussure pense qu'il ne faut pas considérer l'histoire comme une discipline séparée. Ce n'est que dans le cas d'une citation ou lors des extraits sont à inclure dans le procès-verbal de la séance qu'il est possible de trouver des guillemets dans ces textes. Ils ne sont autrement jamais présents dans les registres des séances de la Faculté. Ce dernier cas est justement celui du texte de Saussure.

La présence de ce texte dans les registres et leur caractère officiel donne aussi la possibilité de dater sans problèmes la période durant laquelle Saussure l'a écrit. Le texte a été préparé entre le mois de septembre et le mois d'octobre 1906, probablement après la nomination de la commission pour la reforme des enseignements, donc après le 6 octobre (cf. AUG/RS, 1984/20/91, 6 octobre 1906: 217-218) et la séance à la Faculté du 27 octobre 1906 quand sera lu le texte et puis enregistré dans le registre des séances. 


\section{Document}

Séance du 27 octobre 1906, à 5 h 1/4, Salle du Sénat

Présidence de M. le Prof. Duproix, doyen.

Présents: MM. de Girard, Gourd, Nicole, Muret, de Saussure, Ritter, Adrien

Naville, Oltramare, Gourd, Wuarin, Bouvier, Seitz, Moriaud, Borgeaud, Milhaud.

\section{Réorganisation de l'enseignement de la linguistique}

L'ordre du jour appelle la réorganisation de l'enseignement de la philologie et de la linguistique. Au nom de la Commission nommée dans la précédente séance, et composéede MM. Oltramare, de Saussure et Nicole, M. Nicole rapporte. Les conclusions de la Commission peuvent se résumer ainsi: $1^{\circ}$ Laisser aux professeurs de la Faculté chargé de l'interprétation des textes classiques anciens ou modernes le soin de traiter les sujets qui formaient la matière du cours de philologie ${ }^{31} ; 2^{\circ}$ joindre l'enseignement de la linguistique à celui de la grammaire comparée des langues indoeuropéennes. ${ }^{32}$

Nicole donne ensuite lecture de la note suivante présentée à la commission sur sa demande par M. de Saussure

\section{Programme présenté à la Commission par M. de Saussure}

La Commission m'ayant fait l'honneur de me demander <mon> opinion sur les matières qu'aurait à comprendre un cours de linguistique générale, au cas d'une réorganisation de l'enseignement linguistique à la Faculté, je lui soumets les idées suivantes, en la priant de n'attacher pas plus d'importance que je n'en met moi-même à une énumération détaillée des sujets. ${ }^{33}$

31 À propos de la philologie en Suisse cf. Fryba-Réber (2013).

32 Comme nous venons de le dire, il s'agit donc de joindre la chaire de Wertheimer à celle de Saussure.

33 Saussure soumet des lignes générales pour le cours sans rentrer dans les détails. C'est un trait typique de Saussure de prendre une distance et de faire épreuve de modestie. Nombreux sont les exemples dans la correspondance comme on peut lire dans les échanges avec Antoine Meillet (1866-1936) qui l'invitait à donner une conférence au Collège de France: "Je l'accepte en principe. Je tiens, seulement et au cas où la proposition prendrait corps, à ce que les personnes qui ont à décider, sachent que je ne me reconnais aucune espèce de talent de parole; en sorte que dans une alternative où le Collège aurait à se diriger d'après cette considération, je vous prie de retrancher d'avance mon nom" (Saussure 2014: 466, 12 novembre 1906, Saussure à Meillet). 
En disant que le cours de linguistique aurait à envisager la langue premièrement comme chose qui évolue, ${ }^{34}$ en second lieu dans ce qui caractérise au contraire un état de langue, ${ }^{35}$ on aurait, je crois, tracé par avance un cadre où aucune matière rentrant légitimement dans la Linguistique ne se trouverait oubliée ou inclassée ${ }^{36}$; de sorte qu'en acceptant une telle définition du programme, il y aurait plutôt lieu de prévoir, par la difficulté de donner le même développement à toutes les parties, que certaines d'entre elles ne pourront être traitées, et devrons conduire à un choix, calculé d'après ce qui peut être le plus utile aux étudiants. ${ }^{37}$

De la partie qui est relative à l'état de langue dépendrait d'un part toute la Grammaire générale comme étant le mécanisme de l'expression, ${ }^{38}$ et au même

34 Le secrétaire de la séance recopie la partie du texte soulignée dansle manuscrit de Saussure. 35 La distinction entre la langue pensée comme une chose qui évolue et dans un état correspondrait à la distinction saussurienne entre diachronie et synchronie déjà anticipée par Saussure dans ses leçons parisiennes en 1885 (cf. Joseph 2010) et ensuite reprise durant les premières leçons de son premier cours genevois de Phonétique du grec et du latin (Chidichimo 2010; BGE Ms.fr. 3951/1.1-1.3; CLG/E 3283-3285)où il est question plus particulièrement d'une langue qui est à la fois continuelle et en changement constant. De la même période datent les notes du manuscrit d'ED (BGE AdS 372) où Saussure trace une nette division entre le point de vue dans un état (à ce moment il choisitde ne pas utiliser la synchronie) et le point de vue dans le temps (diachronique; cf. Chidichimo sous presse).

36 On retrouve déjà cette distinction sous laquelledevraient se résumer toutes les disciplines de la linguistique au moins en 1891 dans le cadre des essais de rédaction de ED. Saussure distinguera la phonétique et la sémiologie (cf. supra).

37 La fonction pédagogique est une préoccupation, tout à fait normale dans le cadre d'un cours donné à l'université, et toujours présente chez Saussure, ne serait-ce que par le fait de se trouver à communiquer à une classe souvent composée d'étudiants sans connaissances de linguistique. Son premier objectif en tant que professeur est l'enseignement et non de développer sa propre doctrine sans se soucier de ses obligations pédagogiques. Pour cette raison, en vue de présenter le programme, il annonce d'abord des ajustements durant le cours en prenant en compte les exigences des étudiants. Cf. Joseph (2010) par rapport au cours de Gothique de 1885 à Paris, quand Saussure considérant que la classe est avancée, se lance dans des approfondissements du programme.

38 Pour ce que j'ai pu vérifier, 'Mécanisme de l'expression' ne semble pas être attesté dans les notes saussuriennes, ni dans les textes publiés. Dans Lexique34 on retrouve un passage proche: "système: mécanisme grammatical"; ce passage appartient aux notes en vue du troisième cours (BGE Ms.fr. 3951/23; CLG/E 3338). Dans les notes de Riedlinger du premier cours, on peut lire à propos de la distinction entre phonétique et phonologie:

La linguistique se résolut en études phonologiques: 1) étude du mécanisme de la parole (Sprach-/ Laut-physiologie <Grundzüge der Phonetik; Sievers l'appelle "Phonétique". Les Anglais communément "Lalletik" >) <à> qui l'on peut réserver le nom de "Phonologie," et 2) ces études des lois phonétiques soit de la transformation du son dans l'histoire de la langue, que l'on pourrait comprendre sous la rubrique «Phonétique»." (Saussure 1996: 2)

On retrouvera 'mécanisme de l'expressivité' dans les textes de Bally. Bally l'utilisera tout 
titre la classification des langues d'après le caractère qu'elle emprunte au mécanisme de l'expression (langues flexionnelles ou non-flexionnelles, etc.).

Outre ce qu'on comprend par Grammaire générale, il sera donné ici une vue sur la constitution du mot du coté matériel, comprenant une incursion dans la phonétique physiologique. ${ }^{39}$

La totalité des autres notions sur le mot, comme celle de l'accent, de la quantité, ou de l'importance du mot dans le rythme poétique adopté par chaque langue, trouve encore son expression à propos des états de langues.

L'autre partie du cours, relative aux phénomènes évolutifs, n'est pas, dans mon idée, la plus importante, mais est la plus importante en ce sens que c'est la plus neuve pour les étudiants qui sortent de l'apprentissage pur et simple d'une langue comme le grec et le latin, et la plus propre à leur inspirer de l'intérêt pour ces langues sous un point de vue qu'ils n'avaient pas aperçu. Le cours n'aurait du reste pas à s'arrêter à ces langues, mais à traiter en général:

$1^{\circ}$ De tous les facteurs quiamènent au changement dans la langue Changement phonétique et changement analogique ${ }^{40}$ - Valeur illimité du changement, pouvant créer un <second> état de langue - Illustration par le tableau historique des langues indoeuropéennes, constituant un chapitre à part Différentiation géographique (dialectale) concurrente à la différenciation par le temps. $^{41}$

A chacun de ces parties se rattache tout ce qu'on peut demander secondairement comme par exemple:

Relation de la langue écrite à la langue parlée. - Erreurs qu'a causées l'écriture - rôle des langues littéraires. ${ }^{42}$

Méthode, et erreurs de méthode. - Méthode historique, méthode comparative, méthode reconstructive. ${ }^{43}$

Particulièrement, et comme devant faire l'objet d'une introduction si possible:

particulièrement dans la deuxième édition du recueil d'articles Le langage et la vie de 1926, où il insère le texte Essai sur le mécanisme de l'expressivité linguistique inspiré par une communication qu'il avait fait à la Société de philosophie de Genève en 1925. À propos d'expression et expressivité dans l'École genevoise de linguistique cf. Curea (2015).

39 La "phonétique physiologique" est déjà utilisée par Saussure dans Phonétique, par exemple donc dans les notes parisiennes de 1881-1885, publiées par Marchese (cf. Saussure 1995: 224). 40 Cf. Saussure (1996: 55), où Saussure, après avoir traité du changement phonétique, commence à s'occuper de celui analogique.

41 Cf. Saussure (1996: 107-111).

42 Cf. § 2. du premier cours (Saussure 1996: 3).

43 Saussure traitera la Méthode reconstructive et sa valeur vers la fin du cours (Saussure 1996: 111-120). 
La notion générale de la Langue, ${ }^{44}$ les erreurs à cet égard, et la clarté d'une science linguistique comme études des symboles, ${ }^{45}$ ce qui la place dans le cercle général des sciences sociales.

Un second cours annexe pourrait avoir pour objet:

L'histoire externe des langues d'Europe. C'est à dire l'ensemble des événements politiques, ethnologiques, ou autres, ayant amené le groupement actuel des langues de l'Europe, avec attention particulière aux origines." 46

Après quelques considérations complémentaire qu'expose M. de Saussure, et qui ont trait notamment aux rapports de la linguistique et des sciences sociales en général, M. Muret appuie les conclusions de la Commission. ${ }^{47}$

44 Pour l'emploi de "Langue" en majuscule, cela semble être une des premières fois que Saussure l'utilise de cette façon au moins dans une occasion officielle. On retrouve en effet la notion de langue quand il commence à s'occuper du chapitre 'Linguistique' (cf. Saussure 1996: 27).

45 Saussure recourt à l'emploi du terme symbole plutôt qu'à celui de signe, qui sera utilisé plus tard dans la suite des cours (cf. Lexique: 49). On retrouve une fois ce terme dans les notes du premier cours dans le cadre de l'explication des conséquences du changement phonétique dans le temps dont l'arbitraire du lien entre signifié et signifiant en détermine le caractère de n'être pas prédictible: "Ce caractère des modifications phonétiques d'être incalculables et illimitées vient de la qualité arbitraire du symbole phonétique qui n'a aucun lien avec la signification du mot." (Saussure 1996: 42, Cah. I, Chap. I, § 4). Dans ce cas ce symbole phonétique pourrait se résumer à ce qui sera ensuite le signifiant dans le couple formant le signe, signifié/signifiant, utilisé lors du troisième cours (cf. C: 309; CLG: 99). Si dans le cadre de ce passage du premier cours il faut considérer le terme symbole dans l'acception de symbole phonétique, dans la suite des cours Saussure reviendra sur l'emploi de ce terme en l'abandonnant pour la raison que le terme symbole indique une entité qui n'est jamais complètement arbitraire et en soulignant la distinction avec le signe (Lexique: 49). L'emploi de symbole et la référence au cercle général des sciences sociales, renvoie aux manuscrits de Saussure connus avec le nom de Cahier Whitney (BGE Ms.fr. 3951/10; CLG/E: 3297) et à la définition qu'il donnera en rapportavec la sémiologie, "science qui étudie la langue au sein de la vie sociale" (cf. CLG: 33; Naville 1901; Naville 1914; Gambarara 2007: 254, n.29). Quelques années avant ce premier cours, Saussure s'intéresse déjà de plus près aux recherches sur les légendes germaniques, où le terme symbole revient encore une fois (Saussure 1982). Une recherche sur le terme symbole à travers les publications et les manuscrits de Saussure fera de la clarté sur l'évolution de cette terminologie au sein de la définition du signe linguistique.

46 À ce moment là, Saussure n'a pas encore formulé ce qui sera effectivement le développement du deuxième cours de linguistique général, 1908-1909 (à propos d'une ultérieure annoncede programme du deuxième cours cf. Saussure 1996: 101). En général, la différence entre histoire interne et histoire externe revient dans divers lieux textuels de Saussure et sera traité dans le premier cours dans le cadre d'un Aperçu sur l'histoire interne et externe des langues indoeuropéennes (cf. Saussure 1996: 105 et suiv.). Une référence à l'histoire externe cause du changement se retrouve dans le chapitre dédié au changement phonétique (cf. Saussure 1996: 39-41).

$47 \mathrm{Si}$ on remarque encore le soutien de Muret à son ex professeur, on voit aussi que ces considérations supplémentaires apportées par Saussure n’ont pas étés notées par Milhaud. On peut oser avancer l'hypothèse que ces références continues aux sciences sociales au delà des préoccupations propres à Saussure et inhérentes à l'établissement de la sémiologie en tant que 
M. de Girard marque l'intérêt du programme tracé par M. de Saussure pour les étudiants de sciences sociales.

M. Bouvier informe la Faculté que le Bureau de l'Universitée ${ }^{48}$ se réunira lundi prochain 29 octobre, prendra connaissance des décisions de la Faculté touchant l'enseignement de la linguistique et examinera la question.

Les conclusions de la commission, énoncées plus haut, sont adoptées.

M. Muret propose comme titre du nouvel enseignement: Linguistique générale et grammaire comparé des langues indo-européennes (Approbations)

M. Naville insiste sur l'avantage qu'il y a, pour arriver au but sans recourir à un changement de la loi, à parler d'enseignement, et non de la chaire.

Séance levée à 7 heures.

\section{Abréviations}

AUG/RS Archives de l'Université de Genève/Registre des séances

BGE Bibliothèque de Genève; Ms.fr., Manuscrits Français

AdS Archives de Saussure

C Émile Constantin, Linguistique générale, Cours de M. le Professeur de Saussure, 19101911

CLG/E Cours de linguistique générale, édition critique par Rudolf Engler

CLG/D Cours de linguistique générale, édition critique par Tullio De Mauro

SM Les sources manuscrites du Cours de linguistique générale de F. de Saussure.

\section{Références}

Archives de l'Université de Genève, Registre des Séances du Sénat universitaire.

Archives de l'Université de Genève, Registre des Séances de la Faculté de Lettres et Sciences sociales. [AUG/RS]

Archives de l'Université de Genève, Programmes des cours de l'Université de Genève, années 1891-1906.

Bibliothèque de Genève, Manuscrits Français, 1599, 3951, 3953. [BGE Ms.fr.]

Bibliothèque de Genève, Archives de Saussure, 372. [BGE AdS]

Bally, Charles. 1926. Le langage et la vie. Paris: Payot.

Bergounioux, Gabriel. 2010. La phonologie comme morphologie. In Cristian Bota, Jean-Paul Bronckart \& Ekaterina Bulea (dirs.), Le projet de Ferdinand de Saussure, 105-124. Genève: Droz.

Borgeaud, Charles. 1934. Histoire de l'Université de Genève, vol. 3. Genève: George.

telle, relèvent aussi de raisons institutionnelles, ce cours étant adressé à des étudiants d'une Faculté qui réunissait les licences ès lettres et sciences sociales.

48 Il s'agit du Sénat Académique. 
Bouvier, Bernard. 1907. Discours du Recteur pour l'année 1906. In Archives de l'université de Genève, Séances Officielles, 1907-1912, 28 janvier 1907, 19-29. Genève: Imprimerie Kundig.

Bréal, Michel. 1866. De la forme et de la fonction des mots. Revue des cours littéraires de la France et de l'étranger 29. 65-71.

Chidichimo, Alessandro. 2010. Les premières leçons de Saussure à Genève, 1891: Textes, témoins, manuscrits. Cahiers Ferdinand de Saussure 62. 257-276.

Chidichimo, Alessandro. 2013. Bréal lecteur de Goethe: Un jeu de textes entre réalité et fiction (1898-1911). Cahiers de l'ILSL 37. 187-211.

Chidichimo, Alessandro. en préparation. Index saussurien des Archives de l'Université de Genève.

Chidichimo, Alessandro. sous presse. Saussure et la temporalité: Une recherche terminologique (1881-1891). In Carlos Assunção, Gonçalo Fernandes \& Rolf Kemmler (eds.), History of linguistics 2014: Selected papers from the 13th International Conference on the History of the Language Sciences (ICHoLS XIII), Vila Real, Portugal, 25-29 August 2014. Amsterdam \& Philadelphia: John Benjamins.

Constantin, É. 2005. Linguistique générale, Cours de M. le Professeur de Saussure, 1910-1911. Cahiers Ferdinand de Saussure 58. 83-289.

Curea, Anamaria. 2015. Expression et expressivité: L'école linguistique de Genève de 1900 à 1940. Lyon: ENS Éditions.

Encyclopædia Britannica Online, s. v. "Nicholas Wiseman." http://www.britannica.com/biogra phy/Nicholas-Wiseman (accessed 5 January 2016).

Engler, Rudolf. 1967. Lexique de la terminologie saussurienne. Utrecht: Spectrum. [Lexique]

Fryba-Réber, Anne-Marguerite. 2013. Philologie et linguistique romanes Institutionnalisation des disciplines dans les universités suisses (1872-1945). Louvain: Peeters.

Gambarara, Daniele. 2007. Ordre graphique et ordre théorique: Présentation du Ms. fr. 3951/10 cahier Whitney. Cahiers Ferdinand de Saussure 60. 237-280.

Godel, Robert. 1954. Les sources manuscrites du Cours de linguistique générale de F. de Saussure. Genève: Droz. [SM]

Godel, Robert. 1960. Inventaire des manuscrits de F. de Saussurere mis à la Bibliothèque Publique et Universitaire de Genève. Cahiers Ferdinand de Saussure 17. 5-11.

Joseph, John E. 2008. The attack on Saussure in Le Genevois, December 1912. Cahiers Ferdinand de Saussure 61. 251-281.

Joseph, John E. 2010. Saussure's notes of 1881-1885 on inner speech, linguistic signs and language change. Historiographia Linguistica 37 (1/2). 105-132.

Joseph, John E. 2012. Saussure. Oxford: Oxford University Press.

Le Journal de Genève, 5 mars 1891, 62, n. 55, Chronique locale, p. 5.

Le Journal de Genève, 27 août 1906a, 77, n. 235, Chronique locale, p. 2.

Le Journal de Genève, 5 septembre 1906b, 77, n. 244, Chronique locale, p. 2.

Linda, Markus. 2001. Element einer Semiologie des Hörens und Sprachens. Tübingen: Gunter Narr Verlag.

Mejia, C. \& N. Restrepo-Montoya. 2009. Ferdinand de Saussure, traducteur. Cahiers Ferdinand de Saussure 61. 175-198.

Naville, Adrien. 1914. Faculté de lettres et sciences sociales. In Historique des Facultés 1896-1914. Genève: George.

Naville, Adrien. 1901. Nouvelle classification des sciences, $2^{\text {eme }}$ éd. Paris: F. Alcan. 
Reboul, Fabienne. 2010. La nomination de Saussure à Genève: Une simple formalité? Cahiers Ferdinand de Saussure 62. 217-256.

Saussure, Ferdinand de. 1967/1974. Cours de linguistique générale R. Engler (dir.). Harrasowitz: Wiesbaden. [CLG/E]

Saussure, Ferdinand de. 1982. Les légendes germaniques, A. Marinetti \&M. Meli (dirs.). Este: Editrice Zielo.

Saussure, Ferdinand de. 1995. Phonétique: Il manoscritto di Harvard, Houghton Library bMS Fr 266(8), Maria Pia Marchese (dir.). Firenze: Unipress.

Saussure, Ferdinand de. 1996. Premier cours de linguistique générale, E. Komatsu (dir.). Oxford: Pergamon Press.

Saussure, Ferdinand de. 2001 [1967]. Corso di linguistica generale, T. De Mauro (dir. et trad.). Roma \& Bari: Laterza. [CLG/D]

Saussure, Ferdinand de. 2002. Écrits de linguistique générale, S. Bouquet \& R. Engler (dirs.). Paris: Gallimard. [ELG]

Saussure, Ferdinand de. 2011. Sciences du langage, R. Amacker (dir.). Genève: Droz.

Saussure, Ferdinand de. 2014. Une vie en lettres, C. Mejia (dir.). Paris: Éditions Cécile Defaut.

Vincent, François. 2010. Le premier cours de linguistique générale: Les notes des étudiants dans l'édition et le fonds Engler. Cahiers Ferdinand de Saussure 63. 229-237.

Vincent, François. 2014. Le premier cours de linguistique générale professé par Ferdinand de Saussure à Genève: Cours I et sténographie Caille: Transcriptions et commentaires. Cahiers Ferdinand de Saussure 67. 175-190.

Wertheimer, Joseph. 1865. Les juifs de l'Occident et le judaïsme moderne. Genève: Imprimerie Pfeffer \& Puky.

Wertheimer, Joseph. 1877. La linguistique Discours prononcé le 30 octobre 1877 à l'ouverture du Cours de linguistique. Genève: Impr. Ramboz et Schuchardt.

Wertheimer, Joseph. 1880. Le Talmud, histoire de sa formation. Genève: C. Schuchardt.

Wiseman, Nicolas. 1838. Discours sur les rapports entre la science et la religion révélée. Bruxelles: Société Nationale.

Zwanziger, Ronald. 1981. Joseph Wertheimer. Saussures einziger Amtsvorgänger. In M. Mayrhofer (ed.), Sitzungsberichte des Heidelberg Akademie der Wissenschaft Philosophisch-historische Klasse, 39-43. Heidelberg: Carl Winter. 